\title{
Seminar on Chemotherapy for Malignant Tumours in Childhood
}

\author{
Chairman: IAN S. KIRKLAND (Edinburgh) \\ Moderators: L. K. PICkett (New York), C. E. Koop (Philadelphia), S. E. KeIDAN (Liverpool), \\ G. W. DORMAN (Dallas)
}

The clinical treatment of all forms of cancer rests increasingly on the combination of surgery, radiotherapy, and chemotherapy. Each of these disciplines has its limitations. Radiotherapy can only be applied to a limited field in adequate dosage for cure, except in the treatment of tumours extremely sensitive to X-radiation. Chemotherapy is so far limited by the generally toxic effect on all the cells of the body, as well as on those of the tumour. It would not be unreasonable to hope that a combination of these three would, therefore, offer the best prospects for the patient while awaiting the better understanding of the basic factors controlling the growth of cancer cells.

Of the chemotherapeutic agents available, only three appear at present to have an established place in the treatment of the embryonic tumours of childhood (excluding leukaemias), actinomycin $D$, vincristine sulphate, and cyclophosphamide.

\section{Nephroblastoma}

Over the past 10 years in Scotland, there have been 61 patients registered with nephroblastoma. Only 15 $(25 \%)$ have survived, of whom 3 have received actinomycin D along with other treatment. One, who responded well at first, died after 2 years and 5 months. Two without chemotherapy have survived over 5 years.

L. K. Pickett reported that the best published results before the use of actinomycin $\mathrm{D}$ had come from the Boston Children's Hospital, with an over-all $47 \%$ survival. The critical time of appearance of secondary tumours after the recognition of the primary is within the first year, and $95 \%$ of secondaries will appear within 12-18 months following operation. The best results were in those children who were treated before the age of 1 year, who gave an $85 \%$ survival rate.

The introduction of actinomycin $\mathrm{D}$ has improved the over-all rate of survival, according to Collins' rule, to $67 \%$, and in those treated from the beginning at the Boston Children's Hospital to nearly $85 \%$. As the years pass, however, there are fewer survivors. No other centre has attained this standard, and there is some doubt as to the biological activity of actinomycin $\mathrm{D}$ manufactured in different centres. The policy at present, therefore, is to give actinomycin $\mathrm{D}$ in a dosage close to the limit of tolerance, idiosyncrasy, and response of bone- marrow in the individual, and the response of the bonemarrow may be the most significant index.

S. E. Keidan had treated 18 children suffering from Wilm's tumour with actinomycin $\mathrm{D}$ during the period 1960-1964: 14 had actinomycin D either before, at, or soon after operation, and radiotherapy was also given. Actinomycin D has had an effect on Wilm's tumour, both as a primary form of treatment along with surgery and radiotherapy, and as treatment for those patients in whom the tumour has not been eradicated by the initial treatment. It may delay if not entirely prevent the appearance of metastases.

One patient with bilateral Wilm's tumour was treated by nephrectomy and partial nephrectomy along with actinomycin $\mathrm{D}$ only, as radiotherapy was considered to run too high a risk of radiation nephritis, and is well after 3 years.

C. E. Koop questioned the biological activity of some of the actinomycin $\mathrm{D}$ available. The original drug gave rise to complications when dosage was higher than $75 \mu \mathrm{g}$./ kg., but in the last year there seemed to be less bonemarrow depression, and less skin reaction from additional $X$ rays. He noted the reduction in survival with time in his patients. Whereas originally he had an $82 \%$ survival according to Collins' rule, he now, 7 years later, had an over-all survival of only $59 \%$. He outlined the management of 5 patients with bilateral Wilm's tumour, 3 of whom are still alive. This entailed biopsy to confirm the diagnosis, actinomycin $D$ given as an infusion into each renal artery, post-operative radiotherapy of $1600 \mathrm{R}$ to the larger tumour, and 800 to the smaller tumour. Follow-up intravenous pyelograms and chest radiographs would determine future laparotomy and surgery. Actinomycin D is continued in sixweekly courses, one surviving patient having had 28 such courses.

Because few patients presented initially with lung metastases ( 7 out of the last 75 cases) but developed them usually within three months of operation with radiotherapy ( 19 out of the remaining 68 cases), he suggested that with the combination of actinomycin $\mathrm{D}$, prophylactic irradiation of the lung fields might be considered. $\mathrm{He}$ questioned the value of treating the local area of nephrectomy with $\mathrm{X}$-radiation, unless this were considered, after confirmation of the diagnosis by biopsy, as a 
pre-operative measure along with actinomycin D. $\mathrm{He}$ has carried out this treatment twice with surival up to 14 months.

G. W. Dorman also questioned the value of irradiating the renal bed following removal of an encapsulated tumour, as there can be crippling complications of radiotherapy in young babies. He had a group of 24 patients in whom conventional therapy with surgery, radiotherapy, and actinomycin $\mathrm{D}$ had failed. They were treated with cyclophosphamide with a satisfactory response in 10, with freedom from symptoms and pulmonary metastases for about 12 months. Pulmonary metastases, however, appeared after that time. There was prolongation of life in all 24 patients.

In a group of 13 patients who appeared to be resistant to actinomycin $\mathrm{D}$, he used vincristine sulphate which showed encouraging results in 9. To be effective, a rapid response was necessary, and if there was no response within three weeks of initiating vincristine then the tumours were usually resistant to this drug. $\mathrm{He}$ favoured the combination of actinomycin $D$ and vincristine as initial treatment along with surgery in the patients presenting with pulmonary metastases.

Including those on the Panel, speakers from various centres took part in the discussion, including A. Murray Clarke (Melbourne), D. G. Young (London), J. MacLelland (Edinburgh), A. Jolleys (Manchester). The following conclusions were drawn.

(1) Diagnosis. Even with biopsy, errors in diagnosis could occur in a few instances, which could prove disastrous to the patient. Every diagnostic aid including selective aortogram should, therefore, be employed, especially in the patient whose clinical picture was unusual. Histologically the cystic tumour, and those showing tubular structures, seemed to have a better prognosis than those showing rhabdomyosarcomatous elements.

(2) Surgery. Although surgery is the best method of eradicating the tumour when it is localized, many patients will already have metastatic spread by the time the tumour presents clinically. There may well be a place for pre-operative treatment for a few days, followed by careful extensive surgery. There is a limited place for local pulmonary resection in patients with localized metastatic deposits.

(3) Radiotherapy. This may be most usefully employed both before and after operation along with chemotherapy. Irradiation of the lung fields in place of the tumour bed may well be of great benefit to the patient. If given alone, a dosage of up to $3,500-4,000 \mathrm{R}$. can be given. But in conjunction with actinomycin $\mathrm{D}$, a total of 1,500-3,000 R. should be aimed at. In general, the patient should be given as high a dosage as can be tolerated, the limits being the age of the child, the size of the field, the area involved, and concomitant chemotherapy. There may well be a place for omitting radiotherapy in the very young child with a completely encapsulated tumour.
(4) Chemotherapy. Actinomycin D has increased the survival rate when added to the other conventional methods of treatment. Dosage has varied from 75 to $120 \mu \mathrm{g} . / \mathrm{kg}$. in various centres, and exceptionally to 200 $\mu \mathrm{g} . / \mathrm{kg}$. Owing to the apparent variable biological activity of the drug, actinomycin $\mathrm{D}$ should be given until toxic symptoms appear, particularly depression of the bone-marrow. There is a need for more accurate biological testing of actinomycin D. There is a place for pre-operative treatment with actinomycin $D$, and a continuous intravenous infusion during operation. Intermittent courses of actinomycin $\mathrm{D}$ may well have to be given every 6 weeks at first, and later every 3 months, but so far no limit to the number of post-operative courses has been determined, but they must be continued for at least the first 18 months to 2 years of survival.

When the tumour appears to be resistant to actinomycin D, relief of symptoms and increased survival time can be achieved with vincristine sulphate, in weekly injections of $0 \cdot 025-0 \cdot 1 \mathrm{mg} . / \mathrm{kg}$., or with cyclophosphamide in doses of $5-10 \mathrm{mg} . / \mathrm{kg}$. day for 10 days intravenously, followed orally at about half this dosage.

\section{Neuroblastoma}

C. E. Koop opened the discussion by recounting his experiences of 100 patients with neuroblastoma. There were 36 survivors, 16 of whom had survived more than 10 years, 8 more than 5 years, 6 more than 3 years, and 6 more than 2 years. Most of the survivors were under 1 year of age at the start of treatment. One immediate survivor died 11 years later with multiple bone metastases. Neuroblastoma was divided into six categories depending on the degree of malignancy, but the histology was no key to the expected survival. 36 patients were treated with chemotherapy. Vincristine sulphate was used in 6 patients with only 1 survivor, even though bone and orbital metastases had been present. All drugs seem to have shown some effect in prolonging life, or relieving symptoms temporarily, but there have been no long-term beneficial results after changing from vincristine to other drugs used either alone or in combination.

L. K. Pickett discussed the value of catecholamine levels in the urine in patients with neuroblastoma. Neuroblastomata may have different biochemical compositions despite similar histological appearances. It is necessary, therefore, to measure all the catecholamines and their metabolites in the urine. Both Voorhess in the U.S.A. and Clarke in Melbourne have established normal levels at different age-groups and weights. Of 48 patients with confirmed neural crest tumours, 40 have shown evidence of abnormal catecholamine metabolism. Of the remaining 8,4 were later proved to have a wrong diagnosis, and 4, confirmed as neural crest tumours, were atypical in the age of the patient and site of the tumour.

Metastases have been shown to produce catecholamines, so that serial catecholamine determinations can be used to evaluate success in treatment. Three patients have been re-explored on the basis of raised catecholamines alone, and residual tumours found and treated. 
If catecholamine levels are normal, there is no need to reoperate for residual tumour. They may be in an inoperable site, and patients have survived up to 16 years with residual palpable masses.

So far there had been no reliable consistency between the biological behaviour of the tumour and the pattern of catecholamines, except that dopamine occurred in more malignant tumours.

Treatment should consist of operation, radiotherapy, and cyclophosphamide with or without vincristine. Actinomycin $\mathrm{D}$ seems to have little place in the treatment of neuroblastoma.

G. W. Dorman said that he had treated 19 patients with cyclophosphamide alone, and all responded well but they could not be claimed as cures. Of 13 patients treated with vincristine only, 3 had shown a good response. 9 patients, with 5 good results, had been treated with combined cyclophosphamide and vincristine, as there had been reported a synergism between these two drugs.

J. M. Simister said that it seemed that combined chemotherapy and radiotherapy had better prospects than either treatment alone, and there is some evidence that chemotherapy preceding radiotherapy is better than the reverse order.

The value of vitamin B12 in treatment is unproved.
Other Tumours

I. S. Kirkland outlined the incidence of childhood tumours as listed in the Scottish Tumour Registry during the years 1955-1964 (excluding leukaemia):

Nephroblastoma, 61 with 15 survivors.

Neuroblastoma, 93 with 15 survivors.

Other tumours, 206 with 78 survivors.

Of the 206 other tumours, 89 were intracranial with 37 survivors. The remaining 117 had 41 survivors, and of these 85 were sarcomatous with 28 survivors. Nephroblastoma and neuroblastoma are about equally numerous as abdominal tumours.

Vincristine suphate had been shown to have a temporary effect in reducing the size of locally growing tumours, and might convert an inoperable into an operable growth. Radical surgery still seemed to offer the best hope in this group.

L. K. Pickett mentioned 2 patients recently with rhabdomyosarcoma who responded dramatically to cytosine arabnoside.

S. E. Keidan had used actinomycin D in the treatment of tumours beyond the scope of surgery and radiotherapy. Relief of symptoms, particularly pain, had been the main effect. These tumours included osteogenic sarcoma, synovial sarcoma, rhabdomyosarcoma, and hepatoblastoma. 